Methodology Report

\title{
Methodological Aspects of REM Sleep- Deprivation and Stereological Protocols in the Brain-Stem Respiratory Nuclei
}

\author{
Ali Mohammad Kamali, Ali Noorafshan ${ }^{2,3^{*}}$, Fatemeh Karimi ${ }^{2,3}$, \\ Saied Karbalay-Doust 2,3
}

1Department of Neuroscience, School of Advanced Medical Sciences and Technologies, Shiraz University of Medical Sciences, Shiraz, Iran

${ }^{2}$ Histomorphometry and Stereology Research Center, Shiraz University of Medical Sciences, Shiraz, Iran

${ }^{3}$ Department of Anatomy, School of Medicine,

Shiraz University of Medical Sciences, Shiraz, Iran

Use your device to scan and

read the article online

*Corresponding author:

Ali Noorafshan,

Histomorphometry and

Stereology Research Center,

Shiraz University of Medical

Sciences, Shiraz, Iran

Email: noora@sums.ac.ir

Received: 16.09.2016

Revised: 21.09.2016

Accepted: 29.09.2016

Keywords:

Sleep deprivation,

Respiratory nuclei,

Stereology, Rat

\begin{abstract}
Sleep deprivation (SD) is known to result in a range of neurological, cognitive and physical consequences in chronically-afflicted subjects. The respiratory nuclei of brain-stem tend to play a pivotal part in the regulating sleep function, hence hypothesized to be affected in various types of sleep-related dysfunctions. The purpose of this methodological report is to explain the techniques of REM sleep deprivation and stereology which can be used to consider changes of the quantitative properties of the respiratory nuclei in sleep-deprived rats.
\end{abstract}

doi https://doi.org/10.18869/nrip.jamsat.2.3.283

\section{Introduction}

Sleep is the natural state of mind viewed in human and animals (1). It is known to retain different roles such as development, restoration, learning and memory consolidation $(2,3)$. Sleep problems may lead to obesity, metabolic disorders, and cardiovascular diseases $(4,5)$ as well as behavioral and neurological dysfunctions (6). In today's modern lifestyle, sleep deprivation (SD) is a common phenomenon which can seriously affect the abilities of the subjected individuals (6). The pioneering study on SD was performed on puppies at the end of the 19th century (7) followed by other reports on experimental animal insomnia, mainly in dogs (8) and formal human SD research. In the following years, the dog as animal model for SD was replaced by cat and later by rodents, with the rat being the animal of choice to date (9). One of the typical forms of SD is paradoxical sleep deprivation (PSD) which disrupts rapid eye movement (REM) sleep. 
REM sleep plays an important role in cognitive information processing and memory consolidation. Therefore, PSD may lead to the failure of advanced neural functions, including decision-making, learning and memory $(10,11)$.

REM-SD can be simply created in a rodent model by means of the flower-pot method. After being employed to sleep research in cats (12), the technique was used to deprive rodents from REM sleep. The method is however accompanied by a significant amount of stress (13) which may influence the interpretation of results. In order to minimize the level of stress, the procedure has been modified by employing multiple platforms in a larger tank and through depriving number of animals at the same time (14).

Animals easily move inside the tank, jumping from a platform to another, often interacting with each other, whereby the stress induced by the immobility and social isolation is reduced $(15,16)$.

In some neuroimaging-included investigations on subjects with REM-sleep behavioral disorder (RBD), structural lesions in the brain-stem, which contain the respiratory centers (RC), have been observed (17).

The RCs receive regulatory signals of hormonal, biochemical-, and neural-type and regulate the depth and rate of respiratory function through the related muscles. Damage to RCs may cause respiratory deficiency and ultimately death (18).

In recent decades, the vast majority of studies have been designed with the aim of describing the SD protocol to evaluate the efficacy of drugs in neurodegenerative diseases including rat model of SD leading to cognitive deficits (19).

On the other hand, some investigations focus on morphometric methods which permit structural data to be quantified and thus used in practical research. In this vein, stereology is the threedimensional study of biostructures through the analysis of two-dimensional images (20).

It is a technique and a developing knowledge that uses random, systematic sampling to make quantitative and unbiased data. It is an important and useful tool in many study fields such as neuroanatomy and histology. Stereology is practically based on the geometric principles such as Cavalieri's principle and statistics(20).
Though the alteration in brain structures following SD has the subject of research thus far, studies examining the quantitative features of respiratory nuclei are scant. The present methodological report is intended to describe the methodological aspects of the REM-SD and stereology procedure.

\section{Animals}

Male Sprague-Dawley rats (250-300 g) are retained in standard laboratory conditions, with a $12 \mathrm{~h} / 12 \mathrm{~h}$ light-dark cycle having access to standard lab-blocks and clean water ad libitum. Animals are randomly assigned to three groups, i.e. cage-control, sleep-deprived (SD) and grid-floor. While the control rats receive no intervention, the experiment group undergo sleep deprivation in a multi-platform Plexiglas box. The grid is used as an environmental control. The rats need to be weighed before the procedure and every other day throughout the study.

\section{Sleep deprivation method}

The box floor contains 15 cylindrical platforms, $8.0 \mathrm{~cm}$ high and $6.5 \mathrm{~cm}$ in diameter. The box is topped with $24^{\circ} \mathrm{C}$ water up to $1.0 \mathrm{~cm}$ below the platforms. Animals should have access to free food and water. They are laid on the platform with freedom of movement. When a rat reaches the rapid eye movement (REM) sleep, it falls into the water due to muscle atonia. Animals are placed in the multiple-platform box for $18 \mathrm{~h}$, from $18: 00$ to 12:00 the next day, followed by a $6 \mathrm{~h}$ sleep. The grid makes an environmental control group for the SD which in this group, animals are placed onto a stainless steel-made grid.

The water tanks includes a grid which covers the whole floor, located $9 \mathrm{~cm}$ above it. The rods are fixed $2.3 \mathrm{~cm}$ apart letting the animals to lie down and not fall in the water. The tails may be immersed into the water (21-23) (Figure 1).

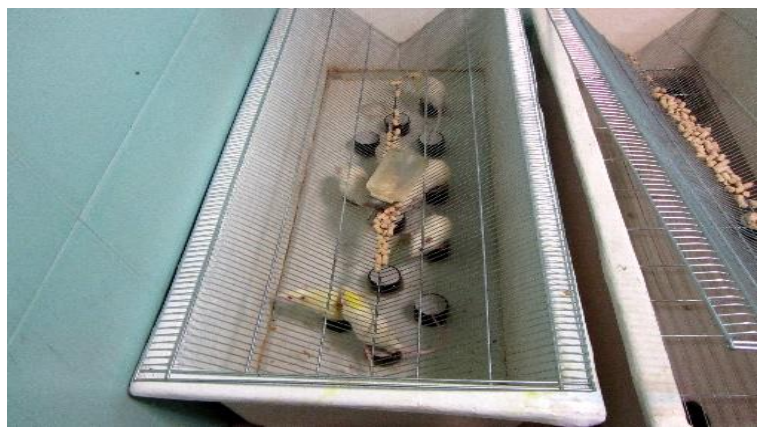

Figure1. Modified multiple platform method-REM sleep deprivation 


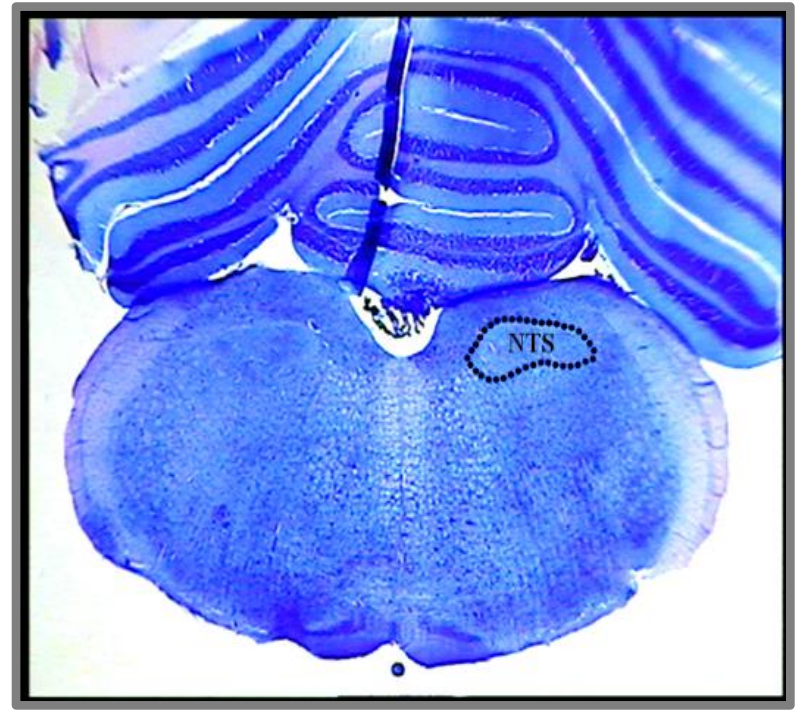

Figure2. Cavalieri method. Respiratory nuclei (including NTS: Nucleus Tractus Solitarius) are found and the sum of the area of each nucleus is estimated using the software.

\section{Histological preparation}

The rats should be sacrificed in 3 weeks after $\mathrm{SD}$. The brain is then removed from the skull and transferred into fixative. Then brainstem is dissected out and prepared for processing, sectioning and staining by means of giemsa stain. Tissue blocks are embedded in paraffin and sectioned coronally on a microtome with a setting of $25 \mu \mathrm{m}$.

\section{- Giemsa staining method}

In order to stain, the sections are heated to $60^{\circ} \mathrm{C}$ for $20 \mathrm{~min}$, dewaxed in xylene for $3 \times 10 \mathrm{~min}$ followed by $10 \mathrm{~min}$ in absolute ethanol, $10 \mathrm{~min}$ in $96 \%$ ethanol, $5 \mathrm{~min}$ in $62 \%$ ethanol, $5 \mathrm{~min}$ in 35\% ethanol, $5 \mathrm{~min}$ in 17\% ethanol and in distilled water for 5 minutes. Then sections are stained for $40 \mathrm{~min}$ with a filtered Giemsa stain stock solution. Ultimately, the sections need to be dehydrated through $0.5 \%$ acetic acid, 96\% ethanol, $5 \mathrm{~min}$ in absolute ethanol and $10 \mathrm{~min}$ in xylene. To evaluate the stereological parameters, one may choose 8-10 sections in a systematic uniform random sampling method (24).

\section{Stereological method}

The classical utilizations of stereology include:

\section{- Estimation of the volume}

Using a stereomicroscope, image from the brainstem sections are assessed according to the

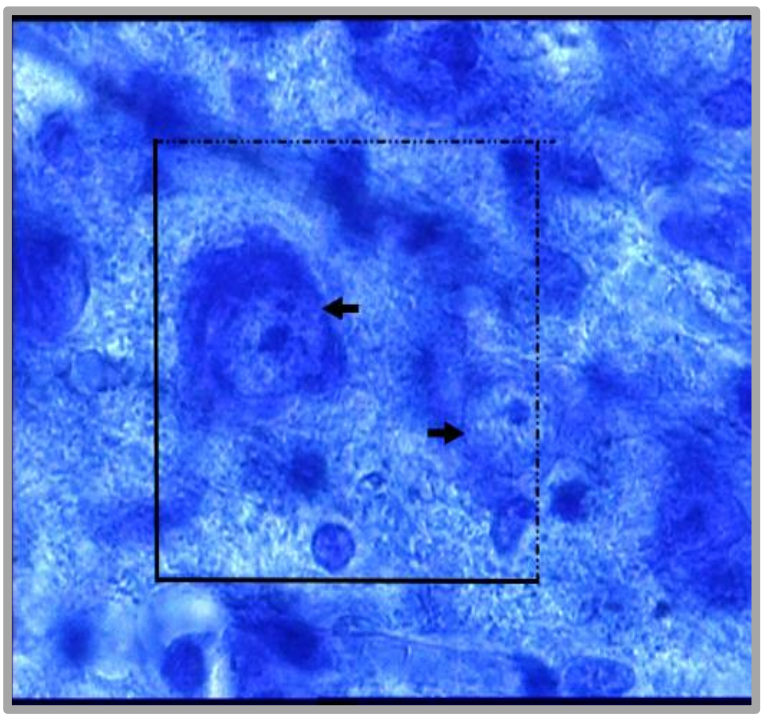

Figure3. Optical dissector method is used to count neurons and the glial cells.

rat brain atlas. The volumes of respiratory nucleus are estimated using the Cavalieri method (25). The images taken from respiratory nuclei are then traced on the sampled sections at the final magnification of $25 \times$. The volume of each structure is estimated using the following formula:

$$
\mathrm{V}(\mathrm{RC})=\sum[\mathrm{A}(\text { sections }) \times \mathrm{d}]
$$

Where ( $\Sigma A)$ (Respiratory nucleus) is the sum of the area of the structure assessed by means of the software and " $d$ " is the interval between the sampled sections (Figure 2).

\section{- Estimation of the cell numbers}

A computer connected to a light microscope and oil immersion lens is employed to calculate the total number of neurons in each respiratory nucleus. The microscopic fields are sampled by moving the microscope with equal intervals, according to the optical dissector method (25). The Optical Dissector is stereological probe to count objects in a thick tissue section. Using a microcator fixed on the stage the z-axis movement of the microscope stage is measured. The unbiased counting frame is used to assess the neurons of the nuclei. Any neuron nucleoli coming into the focus within the sampling box is selected if it is located wholly inside the counting frame and does not touch the boundry lines (left and bottom borders of the frame)(Figure 3 ). The total number of the neurons is then estimated by multiplying the numerical density (Nv) by $\mathrm{V}$ (respiratory nucleus): 


\section{$\mathrm{Nv}$ [Cells/(respiratory nucleus $)]=$ $\left[\left(\sum \mathrm{Q}^{\wedge}-\right) /\left(\sum \mathrm{P} \times \mathrm{a} / \mathrm{f} \times \mathrm{h}\right) \times \mathrm{t} / \mathrm{BA}\right]$}

Where " $\Sigma Q-"$ is the number of the nuclei coming into focus during scanning the " $\mathrm{h}$ " (the height of the dissector) (Figure 1), " $\Sigma \mathrm{P}$ " is the total number of counting frames in all fields, " $a / f$ " is the frame area, " $\mathrm{h}$ " is the height of the dissector , "t" is the mean section thickness measured in every sampled field using the microcator, and "BA" is the block advance of the microtome that is set at $26 \mu \mathrm{m}$ (25).

\section{Conclusion}

There is no single procedure to completely achieve all essential standards to assess the respiratory nucleui. Much of the research have emploed SD as a tool to investigate the neurobiological mechanisms of sleep and sleep loss. A capable method to assess the histomorphological changes in the respiratory nuclei following SD is the stereological analysis.

\section{References}

1. Moreira, C., et al., Arginine metabolism during macrophage autocrine activation and infection with mouse hepatitis virus 3. Immunobiology, 2004. 209(8): p. 585-598.

2. Krueger, J. and F. Obal Jr, Sleep function. Frontiers in bioscience: a journal and virtual library, 2003. 8: p. d511-9.

3. Benington, J.H., Sleep homeostasis and the function of sleep. Sleep, 2000. 23(7): p. 959-966.

4. Grandner, M.A., et al., Sleep disturbance is associated with cardiovascular and metabolic disorders. Journal of sleep research, 2012 21(4): p. 427-433.

5. Spiegel, K., et al., Effects of poor and short sleep on glucose metabolism and obesity risk. Nature Reviews Endocrinology, 2009. 5(5): p. 253-261.

6. Curcio, G., M. Ferrara, and L. De Gennaro, Sleep loss, learning capacity and academic performance. Sleep medicine reviews, 2006. 10(5): p. 323-337.

7. de Manaceine, M., Quelques observations experimentales sur l'influence de l'insomnie absolue. Archives Italiennes de biologie, 1894. 21: p. 322-325.

8. Bentivoglio, M. and G. Grassi-Zucconi, The pioneering experimental studies on sleep deprivation. Sleep, 1997. 20(7): p. 570576.

9. Patrick, G. and J.A. Gilbert, Studies from the psychological laboratory of the University of lowa: On the effects of loss of sleep. Psychological Review, 1896. 3(5): p. 469.

10. Tufik, S., et al., Paradoxical sleep deprivation: neurochemical, hormonal and behavioral alterations. Evidence from 30 years of research. Anais da Academia Brasileira de Ciências, 2009. 81(3): p. 521-538.

11. Machado, R.B., D. Suchecki, and S. Tufik, Sleep homeostasis in rats assessed by a long-term intermittent paradoxical sleep deprivation protocol. Behavioural brain research, 2005. 160(2): p. 356-364.

12. Jouvet, D., P. Vimont, and F. Delorme, Study of selective deprivation of the paradoxal phase of sleep in the cat. Journal de physiologie, 1964. 56: p. 381.

13. Revel, F.G., et al., Rodent models of insomnia: a review of experimental procedures that induce sleep disturbances. Neuroscience \& Biobehavioral Reviews, 2009. 33(6): p. 874-899.

14. Van Hulzen, Z. and A. Coenen, Effects of paradoxical sleep deprivation on two-way avoidance acquisition. Physiology \& behavior, 1982. 29(4): p. 581-587.
15.

Suchecki, D., et al., Increased ACTH and corticosterone secretion induced by different methods of paradoxical sleep deprivation. Journal of sleep research, 1998. 7(4): p. 276-281.

16. Machado, R.B., De bora C. Hipolide, Ana Amelia BeneditoSilva, Sergio Tufik Sleep deprivation induced by the modified multiple platform technique: quantification of sleep loss and recovery. Brain Research, 2004. 1004: p. 45-51.

17. Boeve, B.F., et al., Pathophysiology of REM sleep behaviour disorder and relevance to neurodegenerative disease. Brain, 2007. 130(11): p. 2770-2788.

18. Westfall, D. and T. Westfall, Miscellaneous sympathomimetic agonists. Brunton LL, Chabner BA, Knollmann BC. Goodman \& Gilman's Pharmacological Basis of Therapeutics, 2010.

19. Colavito, V., et al., Experimental sleep deprivation as a tool to test memory deficits in rodents. Frontiers in systems neuroscience, 2013. 7: p. 106.

20. Sundberg, M.D., An introduction to stereological analysis: morphometric techniques for beginning biologists. Chapter, 1992. 3: $p$. 70803-1705.

21. Suchecki, D. and S. Tufik, Social stability attenuates the stress in the modified multiple platform method for paradoxical sleep deprivation in the rat. Physiology \& behavior, 2000. 68(3): p. 309-316.

22. Suchecki, D., B.D. Palma, and S. Tufik, Sleep rebound in animals deprived of paradoxical sleep by the modified multiple platform method. Brain research, 2000. 875(1): p. 14-22.

23. Wang, L., et al., Effects of Wen Dan Tang on insomnia-related anxiety and levels of the brain-gut peptide Ghrelin. Neural regeneration research, 2014. 9(2): p. 205.

24. Samuelsen, G.B., et al., The changing number of cells in the human fetal forebrain and its subdivisions: a stereological analysis. Cerebral Cortex, 2003. 13(2): p. 115-122.

25. Kristiansen, S.L.B. and J.R. Nyengaard, Digital stereology in neuropathology. Apmis, 2012. 120(4): p. 327-340.

\section{Submit your future research: jamsat@sums.ac.ir}

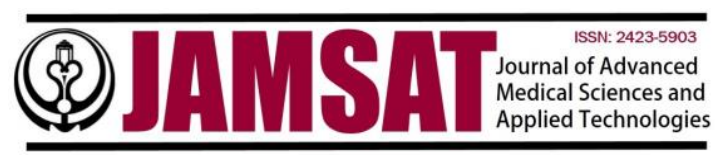

